\title{
Leveraging your content's value
}

\section{Silvio Galea}

has been architecting digital publishing systems and leading teams through web-centric enterprise systems development for over ten years. As Development Manager at Harvard Business School Publishing, Silvio is responsible for driving the development of HBSP's next-generation content-driven applications. Prior to HBSP, Silvio was an independent consultant to various companies with an expertise in transitioning sites to take advantage of Web 2.0 practices. In his prior life, Silvio was the Managing Director of Proxicom's New York technology practice, where he served clients in the publishing, financial, retail and automotive sectors across both the continental United States and Europe.

Keywords: content, content interpreter, content logic, content repository, $x \mathrm{ml}, \mathrm{xml}$ database, enterprise content management

Abstract Content creators are facing the challenge of how to best leverage their content's value. Many are struggling in a world where user-generated content is king and a few niche players who do not have a content-creation background are vying to re-define how people consume content in the digital age. Looking forward, early Web 3.0 speculation is that mature internet users will be seeking premium content and the internet will become a worldwide database requiring content that can be adapted quickly in order to plug seamlessly into new digital formats. This paper introduces the concept of a Content Interpreter, a system that allows content-centric enterprises to leverage their content's value by making it easily available in comprehensible formats. A Content Interpreter is the key to empowering content, especially when working with a large digital archive.

Journal of Digital Asset Management (2007) 3,259-262. doi:10.1057/palgrave.dam.3650101

\section{INTRODUCTION}

Publishers and content-driven enterprises are facing the challenge of how to best leverage their contents' value in the digital age. Many publishers and content creators are struggling to compete in a world where user-generated content is commanding as much attention as professionally generated content, and where companies without publishing or content creation backgrounds are vying to re-define how people consume content.

Some traditional publishers have been late to realize these difficulties - which arose for them with the advent of Web 2.0 - and even more challenges and opportunities are on the horizon. Looking forward to Web 3.0, early speculation is that mature internet users will be seeking premium content, and the internet will become a worldwide database necessitating content that can be adapted quickly and plugged seamlessly into new digital formats.

Faced with current and future challenges, the goal of all publishers and content creators must be to leverage their content's value to its fullest potential through digital distribution, syndication and other content re-purposing strategies.

Today, there are three main hurdles that all content-centric enterprises must overcome in order to thrive:

- preparing and managing content;

- researching and interpreting content to leverage its greatest value;

- deploying content over digital distribution channels.

\section{EMPOWERING YOUR CONTENT}

Business users need to interpret and evaluate their content to best leverage it. To do so, business users need to execute searches across content with an eye to what information might be monetized, how that content might be grouped, packaged or syndicated and how the content will appear across various digital distribution channels.

For such activities to occur effectively across very large content collections, content must be stored in a structured format: broken down into 
contextually defined atomic parts (ie sections, sub-sections, paragraphs) in order to facilitate effective searches. Because of its ability to contextualize and structure content, XML (Extensible Markup Language), as we all know, is the natural format for delivering content over the internet.

Unlike PDFs, Office, Quark, InDesign and other digital formats, the XML format contains all of the data characteristics and structural information needed to aid effective searches and group data effectively. Well-designed XML is contextually self-aware because it not only defines what a specific piece of content is but it also defines its contextual location. For example, XML may show that a particular piece of content is within a sub-section of a section of a larger object and is in the same layer as other objects, etc.

Many content creators are already storing their data in XML, and most content syndication is carried out through an XML format. The bottom line is that the glue that is tying content together on the internet today is XML. Even MS Office 2007 now stores its documents natively in XML as zipped-up collections of XML files under the .docx or . xlsx extension.

\section{MANAGING YOUR CONTENT}

There are two core components to a successful content management strategy.

The first is the versioning, storage and maintenance of the master copy of the files. This is the realm and strength of industry standard Enterprise Content Management Systems (ECMS) and strategies.

Second, content must be made accessible for content managers in a way that allows them to determine the feasibility of a new idea quickly and easily. If a product manager sees an opportunity to provide a collection of content to a partner in a particular format, he should be able to nimbly gauge this opportunity during the time he is on the phone with this partner. If an editor wants to create a collection of assets to give out at a conference, she should be able to browse the content, chunk relevant sub-sections from larger publications and create a customized publication for this specific occasion with rapid ease.

These advanced content browsing and creation capabilities, however, are beyond the capabilities of traditional ECMS. What is needed to meet these needs is a Content Interpreter: a system that sits on an ECMS or a Digital Asset Management System (DAMS) and converts relevant digital content from a large repository into human comprehensible content.

\section{CONTENT INTERPRETER}

In the case of XML, a Content Interpreter is an architecture that consists of an XML database that stores and indexes XML, with a business layer application that provides business intelligence and content transformation capabilities.

These specialized functions are not mature features within ECMS or DAMS. To gain these advantages, it is necessary to look for a thirdparty package to integrate into the ECMS or DAMS. In the market, there exist a number of mature XML Database solutions including but not limited to Mark Logic and the open-source eXist.

The key points of implementing a Content Interpreter are:

1. Integration with the content repository: It is imperative to draw a hard line between the functions that the ECMS and the Content Interpreter are responsible for. For example, the ECMS should "publish" to the Content Interpreter.

2. Scalability: The Content Interpreter should be able to handle an enterprise's full structured content set.

3. Speed: The Content Interpreter must be able to execute queries in real time and can be quickly extended to create new ways of searching and grouping content.

4. Configuration: The Content Interpreter should not be a black box but rather a transparent collection of modules that can be configured to meet new requirements.

5. Lightweight: The Content Interpreter should be relatively portable, and not requisite of an advanced engineering degree to manage and operate.

To meet these five key points of implementing a Content Interpreter, a sophisticated programming language that is simple and lightweight is required. Since the architecture is 
comprised of an XML Database and a business logic layer, the natural choice is XQuery (XML Query Language). XQuery is a natural fit because its inherent capabilities are to execute tasks such as combining, searching and comparing data.

\section{THE CONTENT INTERPRETER'S LAYERS}

The implementation of a Content Interpreter is more an exercise in setting up the appropriate architecture and sticking to a key set of rules as opposed to what tools and technologies are used. Looking at the Content Interpreter from the bottom up, the following outlines its four logical layers:

1. XML or structured content layer - the physical content.

2. ECMS or DAMS layer - the management of the content.

3. XML Database layer - the indexing and searching of the content.

4. Content Interpreter layer - the business application layer.

\section{THE UNDERLYING ASSUMPTION OF XML}

This all assumes that content is in XML. Given the reality of today's content, most of it is not in valid, well-formed XML. It may be in various other digital formats: Quark, InDesign, PDF, MS Office, etc - but this does not mean that it will produce XML that adheres to a Document Type Definition (DTD) that defines the content's structure.

Going about converting an enterprise's content library into XML is unfortunately no easy task. It is an enterprise-wide initiative. One model for implementation is to follow an iterative methodology that minimizes the risk of converting millions of files into XML, only to later realize that one or more core features are missing. The following milestones outline such a process:

- Content definition - define the rules for the content using a DTD. It is recommended to follow an industry standard definition.

- Controlled conversion and prototyping - pick a subset of the content to convert and create prototypical applications on the content. This will expose issues early on.
- Adjust accordingly — address issues within the XML structure and adjust the definition to better meet the enterprise's content needs and requirements.

- Convert - convert the archive in chucks or all at once.

- Standardize - put in place the policies and procedures that dictate how all new content must also be created XML.

At all times, keep it simple and go with industry standards. At the very least, someone else has done the documentation; it is also easier to find talent to supplement and grow an enterprise's teams.

\section{IN PRACTICE}

At Harvard Business School Publishing, the vision of the Content Interpreter is starting to be realized. It started with putting one of HBSP's publications online. At first, the initiative was limited to a scheduled release of new content. To do so in an automated fashion, HBSP decided to start converting the ongoing content of a particular publication to XML. For storage, a source control system was used; as in the early days, this XML was thought of more as code than as content.

As XML started accumulating over time, additional features became possible: backlist access, online collections, full text search, etc. This new promise of automation quickly spread into the back office. Soon, XML was being used to automatically generate reprinted items, syndicated feeds in various structured formats, custom publications and online copies for partners. Content was now able to be semiautomatically published over multiple channels. The functional aspect of this new content format became extremely valuable. Overnight, the amount of XML-related digital assets grew exponentially.

The XML transformation system was dependent on a number of custom-built components. Each of these was developed for a specific purpose. When a new request, such as weighted search, content repurposing and content mash-ups came up, a new black box had to be created. This model quickly hit its limits.

This was quickly addressed by putting in place a native XML Database, on which an open architecture was developed. A robust search 
was added to the database that allowed HBSP to see, cross-reference, analyze and combine content in a human-understandable approach. With the integration into the ECMS, the vision of a Content Interpreter is now being realized.

\section{LOOKING FORWARD}

Web 2.0 has been focused on innovative utilization of the browser and the network, and the evolution of web-based user experience practices and applications. Although content has been a key component, it has not been the driving factor. Web 2.0 is driven by features such as user collaboration, data aggregation, open standards, user-generated content, user participation, bubbly logos and decentralization. One thing this movement did accomplish is to graduate XML to a cross-internet standard. The one thing it did not do was expose enterprise and archived content. There is a mind-boggling amount of content that is waiting to be set free.

Preliminary talk of what constitutes Web 3.0 has to do with "transforming the web into a database" or "World Wide Database." This movement will be centered around content. It is only a matter of time before users migrate to professionally authored, edited and published content. Some of the most valuable content is not yet in a format that will make it accessible by this new movement. Even if an enterprise's content was ready to be delivered in XML, the enterprise must be ready and able to make it available according to standards, methodologies and formats that they are not yet aware of. To make that happen, it is imperative that a Content Interpreter be placed on top of this content. Will your content be ready to be quickly leveraged for these users? 